\title{
Femoral Component Alignment Boundaries for Tibia First Gap Balancing using Digital Tensioning Tool
}

\author{
Edgar A. Wakelin ${ }^{1 *}$, Sami Shalhoub ${ }^{1}$, Jeffrey M. Lawrence ${ }^{2}$, John M. \\ Keggi $^{3}$, Jeffrey H. DeClaire ${ }^{4}$, Amber L. Randall ${ }^{5}$, Corey E. Ponder ${ }^{6}$, Jan \\ Koenig $^{7}$, Christopher Plaskos ${ }^{1}$ \\ ${ }^{1}$ Corin Ltd., Raynham, MA, USA. \\ ${ }^{2}$ Gundersen Health System, Viroqua, WI, USA. \\ ${ }^{3}$ Connecticut Joint Replacement Institute, Hartford, CT, USA. \\ ${ }^{4}$ DeClaire LaMacchia Orthopaedic Institute, Rochester Hills, MI, USA. \\ ${ }^{5}$ Flagstaff Bone and Joint, Flagstaff, AZ, USA. \\ ${ }^{6}$ Oklahoma Sports and Orthopedics Institute, Edmond, OK, USA. \\ ${ }^{7}$ NYU Winthrop University Hospital, Long Island, NY, USA \\ edgar.wakelin@coringroup.com
}

\begin{abstract}
Achieving a balanced knee is a critical aspect of Total Knee Arthroplasty (TKA). Coronal and axial boundaries for femoral component placement to achieve balance however, are not well defined. Our aim is to investigate the effect of femoral component and long leg coronal and axial alignment on patient outcomes when using a tibia-first gap balancing technique.

All surgeries were performed using the OMNIBotics robot-assisted TKA platform and BalanceBot device. A total of 197 patients were prospectively enrolled into this study and received TKA surgery using the OMNIBotics platform and completed 1-year KOOS outcome scores. Femoral component and tibiofemoral alignment were categorized as inliers or outliers in the coronal and axial planes. Knee Injury and Osteoarthritis Outcome Score (KOOS), and University of California at Los Angeles Activity Scale (UCLA) was collected at 1-year post-op.

No significant differences were found between the KOOS subscores or UCLA outcome and femoral coronal or tibiofemoral coronal and axial alignment. Significant differences were found between the KOOS pain and sports sub-scores and femoral axial alignment $(\Delta=5.4, \mathrm{p}=0.007, \Delta=8.3, \mathrm{p}=0.03$ respectively), in which outlier femoral rotation reported higher scores.

Component alignment limits for improved survival and patient outcomes are a source of ongoing debate. The data presented here indicates that when utilizing a tibia-first gap balancing technique, small deviations outside of traditional $\pm 3^{\circ}$ alignment boundaries did
\end{abstract}


not negatively affect KOOS or UCLA outcomes, indicating balance may have a stronger link to patient outcome than alignment.

\section{Introduction}

Achieving a balanced knee is a critical aspect of Total Knee Arthroplasty (TKA). Technology for assessing balance have progressed from laminar spreaders and manual instruments to computer navigated predictive digital balancing [1,2]. Methods of achieving balance depend on surgical philosophy, tibia-first gap balancing favors a neutral tibial resection, followed by a patient specific femoral component placement to achieve extension and flexion balance. Coronal and axial boundaries for femoral component placement using this technique however, are not well defined. Traditional measured resection techniques give acceptable alignment variability as $\pm 3^{\circ}$ from neutral before an increases risk of failure. Literature and alternate alignment strategies however, indicate the failure rate and patient outcomes are patient specific and can extend beyond $\pm 3^{\circ}[3,4]$.

To investigate the effect of femoral component and long leg coronal and axial alignment on patient outcomes when using a tibia-first gap balancing technique.

\section{Methods}

\subsection{Surgical Methods}

All surgeries were performed using the OMNIBotics robot-assisted TKA platform and BalanceBot device. The PCL was routinely resected and APEX implants were used in all cases using either CR femoral component with an Ultra-congruent tibial insert or using PS components. A mixture of tibiafirst gap balancing and femur-first measured resection (targeting mechanical alignment) approaches were performed.

\subsection{Outcome methods and analysis}

A total of 197 patients were prospectively enrolled into this study (Age: $66.7 \pm 8.1$ years, BMI: $31.2 \pm 4.6$ $\mathrm{kg} / \mathrm{m}^{2}$, Gender: $57 \% \mathrm{~F}(112)$, Side: $52 \% \mathrm{~L}(103)$ ) and received TKA surgery using the OMNIBotics platform and completed 1-year KOOS outcome scores. Pre-operative deformity, and tibial and femoral resections were recorded by the OMNIBotics platform. Femoral component alignment was categorized as inliers or outliers in the coronal $\left(>3^{\circ}\right.$ Varus $=$ Outlier, Inlier $\pm 3^{\circ},>3^{\circ}$ Valgus $=$ Outlier $)$ and axial $\left(>1^{\circ}\right.$ Internal $=$ Outlier, Inlier $=1^{\circ}$ internal to $5^{\circ}$ external, $>5^{\circ}$ External $=$ Outlier) planes. Tibiofemoral alignment was also categorized as inliers or outliers in the coronal $\left(>3^{\circ}\right.$ Varus, Neutral $\pm 3^{\circ},>3^{\circ}$ Valgus $)$ and axial $\left(>3^{\circ}\right.$ Internal Rotation, Neutral $1^{\circ}$ internal to $5^{\circ}$ external, $>5^{\circ}$ External) planes. Axial tibiofemoral alignment was calculated as the addition of femoral external rotation and tibial varus. Knee Injury and Osteoarthritis Outcome Score (KOOS), and University of California at Los Angeles Activity Scale (UCLA) was collected at 1-year post-op. Patients were asked to consider the past 7 days as a time frame for the performance of their knee. Wilcoxon rank-sum tests were used to determine the difference between categorized alignment data and patient outcome. All statistical analysis was performed using R 3.5.3.

\section{Results}

The population had a pre-operative deformity of $4.3^{\circ} \pm 5.1^{\circ}$ varus (see Figure 1). A neutral resection was routinely targeted as part of the tibia-first gap balancing or femur first measured resection technique, producing a tibial resection of $0.4^{\circ} \pm 0.9^{\circ}$ varus. The femoral coronal resection was $0.5^{\circ} \pm 2.0^{\circ}$ varus giving a total of 171 knees with inlier alignment (87\%) and 26 knees with outlier alignment (13\%) (see Figure 2). The femoral axial rotation was $2.0^{\circ} \pm 2.7^{\circ}$ external giving a total of 165 knees with inlier 
alignment (84\%) and 32 knees with outlier alignment (16\%) (see Figure 2). The overall post-operative tibio-femoral alignment was $0.9^{\circ} \pm 2.2^{\circ}$ varus giving a total of 157 knees with inlier alignment $(80 \%)$ and 40 knees with outlier alignment (20\%). The tibio-femoral axial alignment $2.4^{\circ} \pm 2.7^{\circ}$ external giving a total of 168 knees with inlier alignment (85\%) and 29 knees with outlier alignment (15\%).

No significant differences were found between the KOOS subscores or UCLA outcome and femoral coronal or tibiofemoral coronal and axial alignment. Significant differences were found between the KOOS pain and sports sub-scores and femoral axial alignment $(\Delta=5.4, \mathrm{p}=0.007, \Delta=8.3, \mathrm{p}=0.03$ respectively), in which outlier femoral rotation reported higher scores (see Figure 2). KOOS questions pertaining to patello-femoral performance were extracted (S4, P3, P6, A1, A2, A3, A5, A7, A9, A10, A11, A13, A15, SP1, AP3) and compared to the categorized groups to investigate how patellofemoral performance is affected by tibiofemoral alignment. No significant differences were found between these questions and femoral coronal or tibiofemoral coronal and axial alignment. Five significant differences were identified when compared to femoral axial rotation: $\mathrm{S} 4(\Delta=0.15, \mathrm{p}=0.015), \mathrm{A} 1(\Delta=0.39, \mathrm{p}=$ $0.003), \mathrm{A} 10(\Delta=0.19, \mathrm{p}=0.049), \mathrm{A} 15(\Delta=0.20, \mathrm{p}=0.038)$ and SP3 $(\Delta=0.51, \mathrm{p}=0.016)$. In all cases the outlier group performed better than the inlier group.

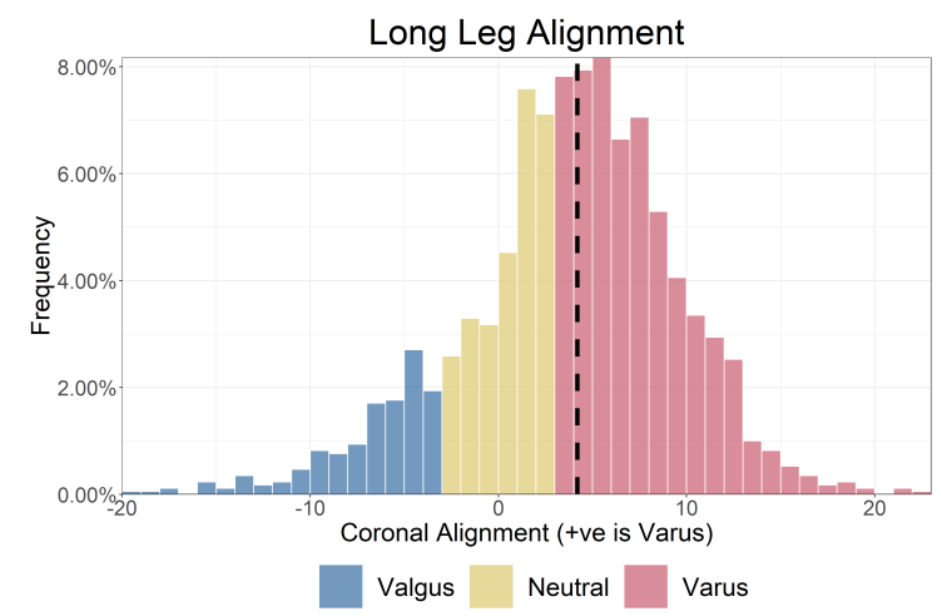

Figure 1 Histogram of Pre-operative Coronal Deformity categorized as Neutral, Varus or Valgus 

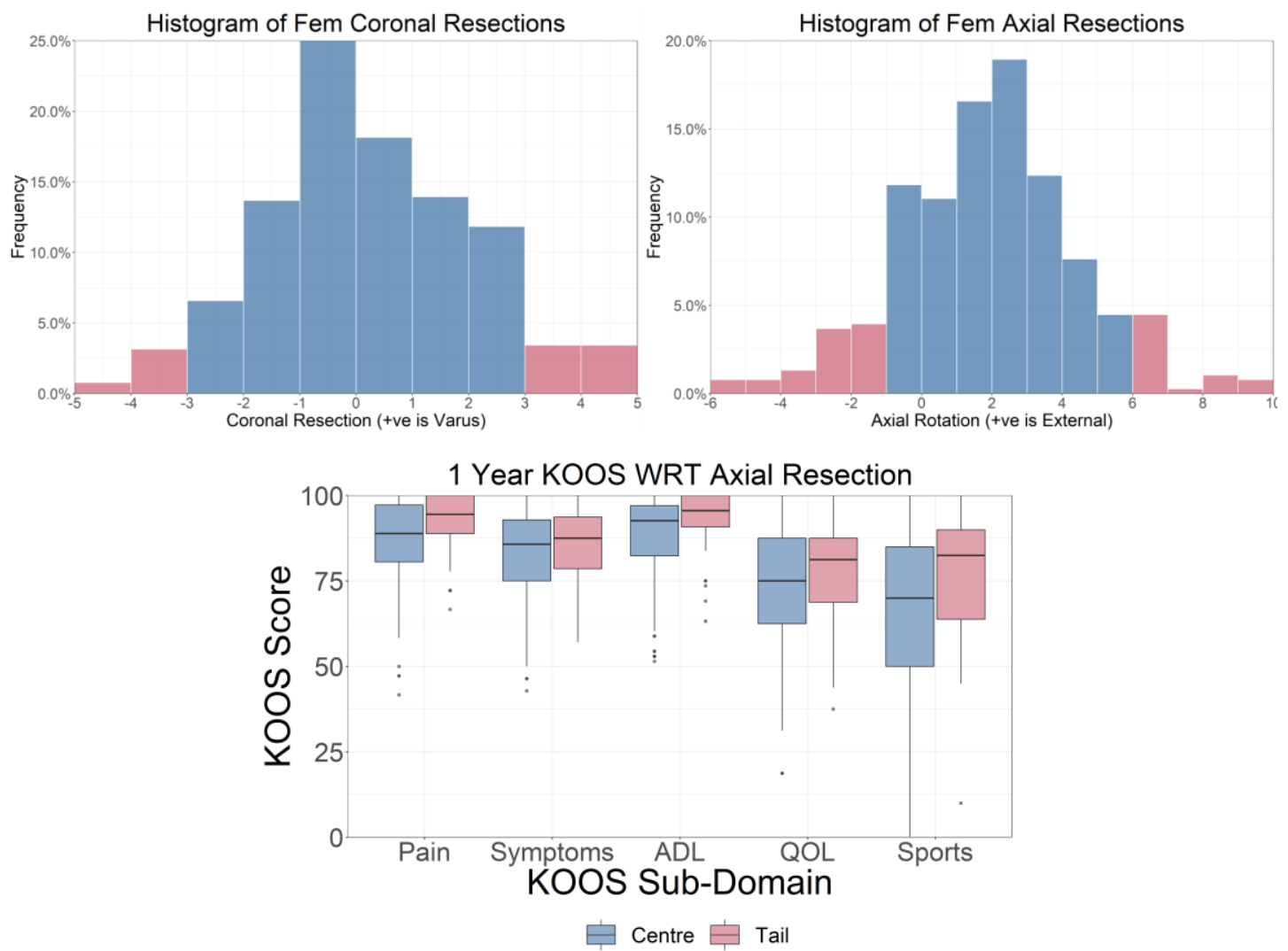

Figure 2 Histograms of Femoral Coronal and Axial Resections. Left: The femoral coronal resection was $0.5^{\circ} \pm 2.0^{\circ}$ varus giving a total of 171 knees with inlier alignment (87\%, blue) and 26 knees with outlier alignment $(13 \%$, rose). Right: The femoral axial rotation was $2.0^{\circ} \pm 2.7^{\circ}$ external giving a total of 165 knees with inlier alignment (84\%, blue) and 32 knees with outlier alignment $(16 \%$, rose). Bottom: KOOS subscores comparing knees with inlier $\left(-1^{\circ}\right.$ to $5^{\circ}$ external rotation, blue-centre) with outlier femoral rotation (rose-tail). Pain and Sports subscores report significantly higher scores in the outlier group compared to the inlier $(\Delta=5.4, p=0.007, \Delta=8.3, p=0.03$ respectively).

\section{Discussion}

Component alignment limits for improved survival and patient outcomes are a source of ongoing debate [5]. The data presented here indicates that when utilizing a tibia-first gap balancing technique, small deviations outside of traditional $\pm 3^{\circ}$ alignment boundaries did not negatively affect KOOS or UCLA outcomes. On the contrary, outlier femoral axial rotation reported improved KOOS pain and sports outcomes, without negatively effecting patella-femoral performance.

Improved outcomes have previously been shown in symmetrically balanced knees [6]. Furthermore, worse outcomes have been associated with additional soft tissue releases to achieve balance. The no difference or improved outcomes shown here may be the result of accepting a wider variation in femoral component placement to achieve a balanced knee without soft tissue release. This data, combined with recent literature, showing similar or improved outcomes using alternate alignment strategies that target joint balance with variable component placement $[7,8]$, indicate balance may have a stronger link to patient outcome than alignment. 
Limitations to this study include: A relatively small number of patients, although data collection is ongoing to investigate the validity of these findings across a wider population; Subdivision of groups into balanced and unbalanced knees in both alignment groups was not possible with the current data set size; Outcomes measured here extend to one year and do not include long term failure rate associated with increased implant wear. Nonetheless, small deviations outside of the traditional $\pm 3^{\circ}$ alignment windows do not appear to effect 1-year KOOS and UCLA outcomes in the population investigated.

\section{References}

1. Golladay, G.J., et al., Are Patients More Satisfied with a Balanced TKA? The Journal of Arthroplasty, 2019.

2. Shalhoub, S., et al., Imageless, robotic-assisted total knee arthroplasty combined with a robotic tensioning system can help predict and achieve accurate postoperative ligament balance. Arthroplasty Today, 2019.

3. Howell, S.M., T.J. Shelton, and M.L. Hull, Implant Survival and Function Ten Years After Kinematically Aligned Total Knee Arthroplasty. The Journal of arthroplasty, 2018.

4. Abdel, M.P., et al., Effect of postoperative mechanical axis alignment on survival and functional outcomes of modern total knee arthroplasties with cement: a concise follow-up at 20 years. JBJS, 2018. 100(6): p. 472-478.

5. Riviere, C., et al., Alignment options for total knee arthroplasty: A systematic review. Orthop Traumatol Surg Res, 2017. 103(7): p. 1047-1056.

6. Gustke, K.A., et al., Primary TKA patients with quantifiably balanced soft-tissue achieve significant clinical gains sooner than unbalanced patients. Advances in orthopedics, 2014. 2014.

7. Woon, J., et al., Outcome of kinematic alignment using patient-specific instrumentation versus mechanical alignment in TKA: a meta-analysis and subgroup analysis of randomised trials. Archives of orthopaedic and trauma surgery, 2018. 138(9): p. 1293-1303.

8. Hommel, H., et al., Small improvements in postoperative outcome with gap balancing technique compared with measured resection in total knee arthroplasty. The open orthopaedics journal, 2017. 11: p. 1236. 